\title{
ALGUNAS REFLEXIONES SOBRE LA PROBLEMÁTICA EN LA EJECUCIÓN DE PROYECTOS HIDROENERGÉTICOS EN EL MARCO DE LA NORMATIVA VIGENTE EN MATERIA DE RECURSOS HÍDRICOS
} A REFLECTION ON THE ISSUE ON THE EXECUTION OF HIDROENERGETIC
PROJECTS WITHIN THE CURRENT WATER RESOURCE REGULATION

\author{
Sandra Lock Benavides* \\ Miranda \& Amado
}

When the law and the competent authorities contradict each other, the execution of projects is discouraged, among the growing legal uncertainty, which occurs with hidro-energetic projects in Peru.

In this article, the author analyzes the current regulation on water resources to then identify and analyze three inconsistencies between what is regulated, what is stated and what actually happens regarding the concept of turbined water, the concept of environmental flow, and the implementation of the Water Availability Leveraging Plan.

KEY WORDS: Water Resource Law, Hidroenergetic Projects, Water rights, Turbined water, Environmental flow, Water Availability Leveraging Plan.
Cuando la legislación y las actuaciones de las autoridades competentes se contradicen, crecen los desincentivos a la ejecución de proyectos en medio de la inseguridad jurídica. Ello es lo que sucede con los proyectos hidro-energéticos en el Perú.

En este artículo, la autora parte de analizar la normativa vigente sobre recursos hídricos para luego identificar y analizar tres contradicciones entre lo normado, lo sancionado y lo que sucede en la realidad respecto del concepto de aguas turbinadas, el concepto de caudal ecológico y la aplicación del Plan De Aprovechamiento de las Disponibilidades Hídricas.

PalabRas Clave: Ley de Recursos Hídricos, Proyectos hidroenergéticos, Derechos de agua, Aguas turbinadas, Caudal ecológico, Plan de Aprovechamiento de las Disponibilidades Hídricas (PADH).

Abogada. Segunda Especialidad en Derecho Ambiental y Recursos Naturales por la Pontificia Universidad Católica del Perú. Asociada del Estudio Miranda \& Amado. Ex-miembro de la Comisión de Publicaciones de la Asociación Civil lus Inter Gentes. Contacto: slock@mafirma.com.pe.

Nota del editor: El presente artículo fue recibido por el Consejo Editorial el 01 de diciembre de 2018, y aceptado por el mismo el 24 de enero de 2019. 


\section{INTRODUCCIÓN}

La actividad de generación eléctrica en el Perú tiene una historia que se remonta muchos años atrás. La electricidad llegó a nuestro país aproximadamente en la penúltima década del siglo XIX y fue hacia el año 1895 que la Empresa Transmisora de Fuerza Eléctrica instaló por primera vez una planta hidroeléctrica en Santa Rosa de la Pampa, ubicada en la margen izquierda del Río Rímac. La primera transmisión fue realizada a las once de la mañana del seis de agosto (Licla, 2012).

El recurso natural esencial que requiere cualquier central hidroeléctrica para operar y desarrollar sus actividades de modo que se produzca la generación de energía es el agua. Así, desde la implementación de la primera central hidroeléctrica, la legislación en materia de recursos hídricos que regula cómo se da el uso de este recurso ha ido variando y adaptándose a distintos estándares ambientales, en algunos casos, cada vez más elevados.

Para tales efectos, desde su creación con fecha 13 de marzo de 2008 mediante el Decreto Legislativo 997, la Autoridad Nacional del Agua (en adelante, "ANA") es el ente rector y máxima autoridad del Sistema Nacional de Gestión de los Recursos Hídricos cuyo fin es dictar las normas y establecer los procedimientos para administrar, conservar, proteger y aprovechar los recursos hídricos de las diferentes cuencas de manera integrada y sostenible. La ANA es un organismo público adscrito al Ministerio de Agricultura y Riego que también ejerce potestad sancionadora en la materia de su competencia, aplicando las sanciones respectivas.

Cabe señalar que, desde el año 2009, contamos como marco regulatorio en materia de aguas con la Ley de Recursos Hídricos, Ley 29338 (en adelante, LRH), y posteriormente, con su reglamento, aprobado en el año 2010, mediante Decreto Supremo 001-2010-AG (en adelante, el “Reglamento").

Adicionalmente a dicha legislación general, la ANA ha emitido diversas resoluciones y normas que contienen lineamientos específicos para cada tema referido al manejo de los recursos hídricos bajo su ámbito de competencia. Sin embargo, como será materia de análisis en el presente artículo, dicha normativa, en ocasiones, ha generado imprecisiones que representan dificultades para la normal ejecución de proyectos de inversión, como es el caso de proyectos hidroenergéticos.

\section{MARCO LEGAL VIGENTE}

Como hemos señalado anteriormente, los derechos de uso del agua en el Perú se rigen por la LRH y su Reglamento. De conformidad con la LRH, los recursos hídricos forman parte del patrimonio nacional del Estado. Por lo tanto, no existe propiedad privada sobre tales recursos.

Siendo este el caso, para utilizar aguas superficiales (incluyendo las aguas marino costeras) o subterráneas para la ejecución de actividades eléctricas, industriales o domésticas, los titulares de tales actividades deberán obtener el correspondiente derecho de uso de agua debidamente otorgado por la ANA, a través de sus órganos desconcentrados ${ }^{1}$.

En ese contexto, la LRH y su Reglamento regulan las siguientes clases de derechos de uso de agua:

a) Licencias de uso de agua, las cuales otorgan a su titular la facultad de usar el recurso hídrico por un plazo indeterminado, con un fin y en un lugar específico, en los términos y condiciones previstos en los dispositivos legales vigentes y en la correspondiente resolución administrativa que la otorga ${ }^{2}{ }^{3}$.

b) Autorizaciones de uso de agua, las cuales otorgan a su titular la facultad de usar un determinado volumen de agua para cubrir exclusivamente las necesidades derivadas o relacionadas directamente con la ejecución de estudios, la ejecución de obras de construcción o el lavado de suelos, según sea el caso, por un plazo no mayor de dos (2) años ${ }^{4}$.

c) Permisos de uso de agua, los cuales pueden ser de los siguientes tipos: (i) títulos habilitantes que se otorgan de manera exclusiva en épocas de superávit hídrico como derechos de duración indeterminada y de ejercicio eventual, que permiten a su titular la

\footnotetext{
Reglamento de Procedimientos Administrativos para el Otorgamiento de Derechos de Uso de Agua y de Autorización de Ejecución de Obras en Fuentes Naturales de Agua aprobada por Resolución Jefatural 007-2015-ANA, publicada el 10 de enero de 2015.

2 Artículo 47 de la Ley 29338, Ley de Recursos Hídricos.

3 De conformidad con el Decreto Supremo 023-2014-MINAGRI, publicado el 27 de diciembre de 2014, que modifica el Reglamento, los procedimientos previos para el otorgamiento de una licencia de uso de agua son los siguientes: (i) autorización para la ejecución de estudios de disponibilidad hídrica; (ii) acreditación de disponibilidad hídrica; y, (iii) autorización de ejecución de obras de aprovechamiento hídrico.

4 Artículo 62 de la Ley 29338, Ley de Recursos Hídricos.
} 
facultad de usar una indeterminada cantidad de agua variable proveniente de una fuente natural mientras dure el estado de superávit hídrico declarado por la ANA; y, (ii) permisos de uso sobre aguas residuales, como derechos de uso de duración indeterminada, que otorgan a su titular la facultad de usar una determinada cantidad de agua variable, proveniente de filtraciones resultantes del ejercicio del derecho de los titulares de licencias de uso de agua 5 .

Considerando lo anterior, las actividades de carácter permanente como la de generación eléctrica requieren de una licencia de uso de agua (ya sea superficial o subterránea) que permita hacer uso de un cierto volumen de recurso hídrico de manera indefinida, siempre que subsista el fin para el que se otorga.

Conforme a la normativa vigente, con la finalidad de obtener una licencia para el uso de agua con fines eléctricos, se deben llevar a cabo los siguientes procedimientos ante la ANA (mediante sus órganos desconcentrados):

(i) Obtención de una autorización para la ejecución de estudios hídricos: Esta autorización no es obligatoria y no otorga a su titular un derecho exclusivo para ejecutar estudios hídricos en un área determinada.

(ii) Acreditación de la disponibilidad hídrica: Con la finalidad de demostrar la disponibilidad de un cierto volumen de agua en una fuente de agua particular y que su uso no afectará los derechos de terceros, los titulares de las actividades hidroenergéticas pueden elegir alternativamente entre las siguientes opciones: (i) la emisión de una resolución que acredita la disponibilidad de un cierto volumen de agua por parte de la ANA (a través de la aprobación de estudios de aprovechamiento hídrico); o, (ii) la emisión de una opinión técnica favorable con respecto a la disponibilidad de agua durante el procedimiento de aprobación del instrumento de gestión ambiental del proyecto hidroeléctrico.

Cabe resaltar que la acreditación de disponibilidad hídrica no otorga un derecho exclusivo a su titular. Asimismo, de confor- midad con el artículo 35 de la LRH, el uso hidroenergético tiene la prioridad más baja para la asignación de derechos de uso agua. De acuerdo con dicha norma, la prioridad es la siguiente: (i) uso primario (es decir, atender las necesidades básicas humanas directamente de la fuente de agua, incluida la preparación de alimentos, higiene personal, uso ceremonial religioso o cultural); (ii) uso doméstico por parte de población u hogares; $y$, finalmente, (iii) uso productivo (incluidos usos industriales, energéticos y mineros).

Adicionalmente, es importante tomar en cuenta que, según el literal b) del artículo 25 de la Ley de Concesiones Eléctricas, aprobada por Decreto Ley 25844, es un requisito de la solicitud para obtener una concesión definitiva de generación eléctrica otorgada por el Ministerio de Energía y Minas (en adelante, MEM) que se presente una autorización para el uso de los recursos naturales de propiedad del Estado.

En tal sentido, para el caso de proyectos hidroenergéticos, este requisito se cumpliría con la obtención de la acreditación de la disponibilidad hídrica. Así, mediante dicha acreditación para determinado proyecto hidroenergético, el titular se encuentra facultado para solicitar la concesión definitiva de generación eléctrica. Una vez obtenido dicho título habilitante, la ANA no podrá aprobar otras acreditaciones de disponibilidad hídrica en la misma área en relación con el volumen disponible de agua aprobado en la resolución correspondiente ${ }^{6}$.

(iii) Obtención de una autorización para la ejecución de obras hidráulicas: Esta autorización otorga el derecho de llevar a cabo la construcción de la infraestructura necesaria para el uso del agua. La autorización requiere la aprobación previa del instrumento de gestión ambiental para el proyecto hidroenergético (como un Estudio de Impacto Ambiental) y evidencia de que el titular tiene los derechos necesarios para el acceso al área en la que se realizarán las obras.

(iv) Obtención de la licencia de uso de agua: Una vez finalizados los procedimientos descritos en los numerales (ii) y (iii), la ANA puede

Artículos 58 y 59 de la Ley 29338, Ley de Recursos Hídricos.

6 Mediante Decreto Supremo 041-2011-EM, se establecieron disposiciones relativas al cumplimiento de requisitos para solicitar concesión temporal, concesión definitiva de generación, otorgamiento de autorización de ejecución de obras, autorizaciones de uso de agua para obras o estudios de generación eléctrica, otorgamiento y extinción de licencias de uso de agua otorgada a titulares de derechos eléctricos. 
otorgar una licencia de uso de agua. La licencia autoriza a su titular el uso de un determinado volumen de agua (establecido en la licencia) de una fuente de agua específica para la ejecución de actividades hidroenergéticas por un período de tiempo indefinido.

En vista de lo antes expuesto, todo titular de un proyecto hidroenergético requiere de la obtención de una licencia de uso de agua con fines eléctricos para realizar sus operaciones. Luego de obtenido este título habilitante, el titular del mismo se encontrará sujeto a la normativa vigente en materia de recursos hídricos y deberá cumplir con lo establecido en la LRH y su Reglamento, así como la demás normativa emitida por la ANA que establece otras obligaciones con la finalidad de regular el uso sostenible del agua.

\section{ALGUNOS ASPECTOS CONTROVERSIALES IDENTIFICADOS EN LA REGULACIÓN EN MA- TERIA DE RECURSOS HÍDRICOS PARA LA EJE- CUCIÓN DE PROYECTOS HIDROELÉCTRICOS}

En línea con lo antes expuesto, si bien tanto la LRH y su Reglamento como las normas emitidas por la ANA han ordenado el marco regulatorio vigente $y$ han establecido criterios que deben ser cumplidos tanto por las autoridades competentes en materia de aguas como por los titulares de derechos para el uso del agua (como sería una empresa hidroeléctrica), en la actualidad existen aún algunos vacíos y/o inconsistencias en dicha normativa que pueden generar que, durante la ejecución de proyectos hidroenergéticos, se produzcan trabas que impidan un regular desarrollo de las actividades de los titulares de estos proyectos.

Es importante tomar en cuenta que, si bien la ANA es el ente rector del Sistema Nacional de Gestión de los Recursos Hídricos, otras autoridades administrativas también tienen competencia para supervisar y fiscalizar el cumplimiento de la normativa ambiental y garantizar el uso sostenible de los recursos naturales -como es el caso del agua- por parte de los titulares de proyectos hidroenergéticos.

Principalmente, cabe resaltar el caso del Organismo de Evaluación y Fiscalización Ambiental (en adelante, "OEFA"), el cual es un organismo público técnico especializado que se encuentra adscrito al Ministerio del Ambiente y es el ente rector del Sistema de Evaluación y Fiscalización Ambiental, cuya función principal es la fiscalización, supervisión, evaluación, control y sanción en materia ambiental, así como la aplicación de incentivos. El OEFA ejerce las funciones previstas en el Decreto Legislativo 1013 y la Ley del Sistema Nacional de Evaluación y Fiscalización Ambiental, Ley 293257.

En ese contexto, mediante el presente artículo se analizarán tres (3) aspectos controversiales que se han identificado en la legislación vigente en materia de recursos hídricos, así como en los pronunciamientos del OEFA que se encuentran poco claros o requieren un mayor desarrollo y precisión para evitar que se produzcan contradicciones que impliquen un desincentivo para la ejecución de proyectos hidroenergéticos en el Perú.

\section{A. Sobre la definición de "aguas turbinadas"}

El término aguas turbinadas resulta bastante común cuando hacemos referencia a proyectos hidroeléctricos y se refiere, bajo un entender coloquial, a aquellas aguas que han sido aprovechadas para la generación de energía eléctrica a través de su paso por las turbinas de una central hidroeléctrica.

No obstante, este concepto ha generado controversia debido a la naturaleza que tendrían las aguas turbinadas; es decir, si constituyen aguas residuales y/o efluentes en los términos de las normas vigentes en materia de recursos hídricos o si, por el contrario, considerando sus características específicas, no lo son.

Esto último tiene relevancia práctica pues en caso de considerarse las aguas turbinadas como aguas residuales, los titulares de proyectos hidroeléctricos requerirían una autorización de vertimiento de aguas residuales emitida por la Dirección de Gestión de Calidad de los Recursos Hídricos (en adelante, "DGCRH") de la ANA previamente a verterlas en un cuerpo natural de agua, y deberían cumplir con otras obligaciones ambientales como la ejecución de monitoreos periódicos.

Si bien las normas vigentes no eran claras respecto a la obligación de contar con una autorización de vertimiento de aguas residuales tratadas para las aguas turbinadas generadas por las centrales hidroeléctricas, mediante el Oficio 513-2013-ANA-DGCRH, de fecha 28 de agosto de 2013, la DGCRH de la ANA pareció resolver finalmente esta disyuntiva.

\footnotetext{
De conformidad con la Resolución de Consejo Directivo 001-2011-OEFA-CD, que aprobó los aspectos objeto de la transferencia de las funciones de supervisión, fiscalización y sanción ambiental en materia de hidrocarburos en general y electricidad entre el Organismo Supervisor de la Inversión en Energía y Minería - OSINERGMIN y OEFA, desde el 4 de marzo de 2011 el OEFA es la autoridad competente a cargo de la regulación, supervisión e imposición de sanciones a las empresas hidroeléctricas en materia ambiental.
} 
A través del Oficio antes mencionado esta dirección respondió a la consulta planteada por el OEFA referida a si correspondía a las centrales hidroeléctricas obtener una autorización de vertimiento para aguas turbinadas o algún otro permiso, de ser el caso. La DGCRH señala que, según la definición establecida en el artículo 131 del Reglamento, son aguas residuales aquellas cuyas características originales han sido modificadas por actividades antropogénicas que tengan que ser vertidas a un cuerpo natural de agua o reusadas, y que, por sus características de calidad, requieran de un tratamiento previo.

Sobre la base de dicha definición, la DGCRH señala que "las aguas captadas de una fuente natural de agua para generar energía eléctrica no sufren modificaciones en sus características originales, pues ingresan a las turbinas y posteriormente regresan al cuerpo receptor". En tal sentido, dichas aguas no son consideradas aguas residuales y por lo tanto no requieren autorización de vertimiento de aguas residuales tratadas.

En línea con lo antes expuesto, no se requeriría obtener una autorización de vertimiento de aguas residuales tratadas para las aguas turbinadas generadas durante la operación de una central hidroeléctrica.

Sin perjuicio de ello, en la sección 6 denominada "Criterios Sustantivos Emitidos en el Subsector Electricidad" del Capítulo II del libro "Principales criterios resolutivos adoptados en los procedimientos administrativos sancionadores del Organismo de Evaluación y Fiscalización Ambiental (OEFA)" elaborado por esta entidad, se establece que las aguas turbinadas constituyen efluentes líquidos conforme con la normativa eléctrica, toda vez que son flujos descargados al ambiente que provienen de las operaciones de generación, transmisión y distribución eléctrica (OEFA, 2016).

Al respecto, el OEFA señala que la Resolución Directoral 008-97-EM/DGAA, que aprobó los Niveles Máximos Permisibles para Efluentes Líquidos Producto de las Actividades de Generación, Transmisión y Distribución de Energía Eléctrica, define a los efluentes líquidos generados específicamente durante las actividades de electricidad como los flujos descargados al ambiente, que provienen de las operaciones de generación, transmisión y distribución de energía eléctrica.

Por ello, se menciona que las aguas que pasan por las turbinas (aguas turbinadas) constituyen efluentes líquidos, en tanto son utilizadas para la generación de energía eléctrica, pasando por diferentes etapas antes de su descarga a la fuente de agua de la cual fueron obtenidas.
Asimismo, según lo señalado por el OEFA, si bien las aguas turbinadas no son sometidas a tratamientos químicos previos a su descarga, pueden sufrir alteraciones en sus características físico-químicas debido a que durante su paso por las turbinas no solo se altera el nivel de temperatura, sino que también se mezclan con los sedimentos depositados en el desarenador y existe el riesgo de que se contaminen con los aceites y grasas de las turbinas por inadecuado mantenimiento de las mismas, lo que puede alterar la calidad del recurso hídrico.

Al ser consideradas las aguas turbinadas como efluentes, el OEFA ha señalado que corresponde la ejecución de la obligación por parte de los titulares de concesiones y autorizaciones eléctricas de realizar los muestreos correspondientes y análisis químicos con una frecuencia mensual, tal como establece la Resolución Directoral 008-97-EM/DGAA, que aprobó los Niveles Máximos Permisibles para Efluentes Líquidos producto de las Actividades de Generación, Transmisión y Distribución de Energía Eléctrica.

Este criterio -confirmado tanto por la Dirección de Fiscalización y Aplicación de Incentivos como por el Tribunal de Fiscalización Ambiental del OEFAha sido adoptado en el procedimiento administrativo sancionador seguido con el Expediente 5082013-OEFA/DFSAI/PAS, en el cual se interpuso un recurso de reconsideración contra una resolución directoral que declaraba la responsabilidad de una empresa por no realizar el monitoreo de efluentes líquidos en un punto de control de monitoreo.

En ese contexto, el oficializado "Glosario de Términos sobre Recursos Hídricos", aprobado mediante Resolución Jefatural 180-2016-ANA (en adelante, el Glosario), define "aguas turbinadas" como

Aquellas procedentes de un cuerpo de agua que han sido aprovechadas para la generación hidroeléctrica que no requieren de una modificación de su estado natural, ni la adición de elementos que alteren dicho estado. En atención a sus características no son consideradas aguas residuales industriales. [El énfasis es nuestro].

Por otro lado, el Glosario define efluente como "aquel líquido o agua residual previamente tratada proveniente de actividades antropogénicas que pueden ser vertidas a un recurso hídrico o reusadas". Siendo ello así, para que las aguas turbinadas puedan ser consideradas efluentes previamente tendrían que ser consideradas aguas residuales; es decir, cuyas características originales han sido modificadas debido a actividades antropogénicas. Como se ha señalado en párrafos anteriores, de 
conformidad con las definiciones establecidas por las normas vigentes en materia de recursos hídricos y la posición adoptada por la DGCRH, este no sería el caso de las aguas turbinadas.

Ahora bien, es importante tomar en cuenta que el último proyecto de Reglamento de Protección Ambiental en las Actividades Eléctricas, que fue pre publicado por el MEM con fecha 25 de enero de 2018 en el Diario Oficial El Peruano mediante Resolución Ministerial 031-2018-MEM/DM, ha precisado que las aguas turbinadas que provienen de la operación de una central hidroeléctrica no son consideradas como agua residual ni como efluente. Esto último en línea de lo señalado por la ANA en el Glosario. Sin perjuicio de ello, el proyecto establece que dichas aguas deberán ser monitoreadas con una periodicidad semestral, de acuerdo a las condiciones establecidas en el estudio ambiental.

En tal sentido, el sector energía representado por el MEM está excluyendo expresamente las aguas turbinadas de ser consideradas como efluentes líquidos generados durante las actividades eléctricas. Ello pues establece que las aguas turbinadas son una excepción al concepto de efluentes contenido en la Resolución Directoral 008-97-EM/ DGAA, que los define de manera general como los flujos descargados al ambiente que provienen de las operaciones de generación, transmisión y distribución de energía eléctrica.

Así, al no ser aguas residuales ni efluentes, a las aguas turbinadas no se les aplicarían las condiciones de los muestreos y análisis químicos señalados en la Resolución Directoral 008-97-EM/DGAA, sino únicamente aquellas establecidas en el estudio ambiental aprobado para el caso concreto.

En atención a lo anterior, consideramos que la definición de aguas turbinadas como efluentes que ha señalado el OEFA en el libro "Principales criterios resolutivos adoptados en los procedimientos administrativos sancionadores del Organismo de Evaluación y Fiscalización Ambiental (OEFA)" y la posición de dicha entidad en el sentido de que es necesario efectuar muestreos y/o monitoreos de estas aguas en virtud de la Resolución Directoral 008-97-EM/DGAA, contradeciría tanto la definición adoptada por la ANA (autoridad competente con competencia exclusiva en materia de recursos hídricos) como por el MEM.
Esta contradicción en la definición del término aguas turbinadas y su categorización como efluentes líquidos podría generar confusiones a los titulares de proyectos hidroenergéticos respecto a las obligaciones ambientales que les resultarían aplicables en relación a dichas aguas, como son la obtención de una autorización de vertimiento de aguas residuales tratadas o la ejecución de muestreos y análisis químicos mensuales, entre otras.

Por todo lo antes expuesto, consideramos que resulta de vital importancia que las entidades competentes sobre esta materia alineen criterios en relación al tratamiento que se les debe dar a las aguas turbinadas para que de esa manera no se generen discordancias como las antes descritas.

Así, podría brindarse una definición única de aguas turbinadas para los titulares de proyectos hidroenergéticos y se tendrían claras las obligaciones ambientales fiscalizables que resultan exigibles por cada entidad competente. Lo anterior daría mayor seguridad jurídica y predictibilidad y evitaría que se inicien procedimientos administrativos sancionadores innecesarios por la falta de claridad de las normas ambientales vigentes.

\section{B. Sobre el concepto de "caudal ecológico"}

El concepto de caudal ecológico -según las normas actualmente vigentes en materia de recursos hídricos- se refiere a "aquel volumen de agua que se debe mantener en las fuentes naturales de agua para la protección o conservación de los ecosistemas involucrados, la estética del paisaje u otros aspectos de interés científico o cultural"8.

Así, "los caudales ecológicos se mantienen permanentemente en su fuente natural, constituyendo una restricción que se impone con carácter general a todos los usuarios de la cuenca, quienes no podrán aprovecharlos bajo ninguna modalidad para un uso consuntivo"'.

Siendo este el caso, la LRH, promulgada en el año 2009, y el Reglamento, aprobado en el año 2010, son las primeras normas que regularon los alcances del concepto de caudal ecológico según lo señalado en los párrafos precedentes, no existiendo anteriormente ningún antecedente o dispositivo legal que lo definiera de esta manera.

$8 \quad$ Numeral 153.1 del Artículo 153 del Reglamento de la Ley de Recursos Hídricos, Ley 29338, aprobado por Decreto Supremo 001-2010-AG.

$9 \quad$ Numeral 153.3 del Artículo 153 del Reglamento de la Ley de Recursos Hídricos, Ley 29338, aprobado por Decreto Supremo 001-2010-AG. 
Sin perjuicio de lo anterior, desde antes de la existencia de este concepto de caudal ecológico, las empresas hidroeléctricas hacían referencia en los instrumentos de gestión ambiental (en adelante, "IGA") aprobados para sus actividades al respeto de un caudal mínimo o incluso caudal ecológico en fuentes naturales de agua. Sin embargo, estos términos al encontrarse en IGA anteriores a la emisión de la LRH y el Reglamento evidentemente no abarcaban todos los componentes del concepto de caudal ecológico vigente a la fecha.

No obstante, cuando el OEFA ha fiscalizado el cumplimiento de compromisos ambientales que mencionan estos términos de caudal mínimo o caudal ecológico, erróneamente ha aplicado "de manera referencial" criterios establecidos en normas vigentes en materia de caudal ecológico, como es el caso de la Metodología para determinar caudales ecológicos, aprobada mediante Resolución Jefatural 154-2016-ANA, publicada con fecha 17 de junio de 2016 (en adelante, la "Metodología").

Dicha aplicación imprecisa realizada por el OEFA ha sido identificada en el marco de los Expedientes 615-2013-OEFA/DFSAI/PAS y 598-2014-OEFA/DFSAl/PAS seguidos contra dos empresas titulares de proyectos hidroenergéticos.

Sobre el particular, cabe señalar que en los IGA aprobados a fines de los años 90 para la ejecución de sus actividades eléctricas, dichas empresas se habían comprometido en el primer caso a respetar un caudal ecológico, mientras que en el segundo caso a mantener un caudal mínimo. En ambos supuestos con la finalidad de preservar el hábitat acuático y a los organismos que se desarrollaban en las fuentes naturales de agua donde se ejecutaban sus proyectos hidroenergéticos. Sin embargo, en ningún momento se habían considerado variables adicionales como la protección del paisaje $u$ otros temas culturales y/o científicos.

A pesar de ello, el OEFA interpretó en ambos expedientes que la referencia a caudal mínimo o caudal ecológico en los IGA aprobados a favor de dichas empresas debía considerar todas las variables de las normas hídricas vigentes, por lo que sí era posible la aplicación tanto de la LRH y su Reglamento como de la Metodología -de manera referencial- para establecer criterios como la determinación del tramo donde debe ser evaluado el cumplimiento del caudal ecológico o el contenido que debe tener un estudio para la determinación del caudal ecológico.
Cabe resaltar que lo expuesto por el OEFA en el marco de estos procedimientos administrativos sancionadores es incorrecto pues el término caudal ecológico definido en la LRH y su Reglamento no es aplicable -en ningún supuesto- para las actividades eléctricas que realizaban las empresas hidroenergéticas antes de la entrada en vigencia de estas normas.

La Ley General de Aguas, aprobada mediante Decreto Ley 17752 (en adelante, "Ley de Aguas") -norma que precedía a la actual LRH y en cuya vigencia se aprobaron los IGA de las empresas antes mencionadas-, no establecía un concepto de caudal ecológico. El artículo 47 de la Ley de Aguas incluía una referencia general a la determinación por parte del Ministerio de Agricultura de un caudal mínimo debajo del cual un Valle o Distrito de Riego podía ser declarado en estado de emergencia por escasez.

Sin embargo, los caudales mínimos referidos en la Ley de Aguas no llegaron a ser regulados por el Ministerio de Agricultura. Por lo tanto, durante toda la vigencia de la Ley de Aguas no existieron caudales mínimos determinados que sirvieran de referencia durante la elaboración de los estudios de aprovechamiento hídrico, estudios hidrológicos ni los estudios ambientales que eran sometidos a evaluación en los diferentes sectores productivos.

Es recién a partir del año 2009 que la LRH y el Reglamento incorporaron el concepto de caudal ecológico, entendido bajo los términos antes mencionados. En tal sentido, la LRH señala como uno de los presupuestos para el otorgamiento de un derecho de uso de agua "que la fuente de agua sobre la que se solicita el aprovechamiento tenga un volumen de agua disponible que asegure los caudales ecológicos"10.

El numeral 2 del artículo 153 del Reglamento señala que:

En cumplimiento del principio de sostenibilidad, la Autoridad Nacional del Agua, en coordinación con el Ministerio del Ambiente, establecerá los caudales de agua necesarios que deban circular por los diferentes cursos de agua, así como, los volúmenes necesarios que deban encontrarse en los cuerpos de agua, para asegurar la conservación, preservación y mantenimiento de los ecosistemas acuáticos estacionales y permanentes.

10 Numeral 2 del artículo 53 de la Ley 29338, Ley de Recursos Hídricos. 
En tal sentido, resulta evidente que es a partir de la vigencia de la LRH (en el año 2009) que se incorpora por primera vez en la normativa en materia de aguas el concepto de caudal ecológico; y, por lo tanto, que los titulares de cualquier derecho de uso de agua pasan a estar obligados a tomarlo como referencia en los planes de aprovechamiento hídrico para el desarrollo de sus actividades.

De esta manera, durante la época en la cual las empresas hidroeléctricas a las que se hizo referencia en párrafos anteriores elaboraron y obtuvieron la aprobación de sus IGA señalando que mantendrían un caudal mínimo o incluso un caudal ecológico, no existía el concepto de caudal ecológico vigente ni metodologías aprobadas para su determinación. Por ello, no puede interpretarse que los IGA de estas empresas hicieron referencia al concepto de caudal ecológico contenido en la LRH y el Reglamento. Lo contrario implicaría una vedada aplicación retroactiva de la LRH.

Ahora bien, en relación a la Metodología, es necesario mencionar que el numeral 5.1 del artículo 5 de esta norma ha señalado de manera acertada que "(I)a determinación de caudales ecológicos efectuada a mérito de la presente resolución no afecta el ejercicio de los derechos de uso de agua otorgados con anterioridad a su entrada en vigencia". [El énfasis es nuestro].

En tal sentido, la Metodología solo es aplicable para la elaboración de nuevos IGA y no para aquellos aprobados durante la vigencia de la Ley de Aguas, no siendo exigible ni aplicable en los casos materia de los Expedientes. 615-2013-OEFA/DFSAl/PAS y 598-2014-OEFA/DFSAI/PAS.

Tal como hemos señalado anteriormente, durante los procedimientos administrativos sancionadores seguidos con dichos expedientes, tanto la Subdirección de Instrucción e Investigación (actualmente, la Subdirección de Fiscalización en Energía y Minas) como la Dirección de Fiscalización, Sanción y Aplicación de Incentivos, actualmente la Dirección de Fiscalización y Aplicación de Incentivos (en adelante, "DFAI") del OEFA aplicaron la Metodología para determinar el área de influencia del proyecto en la cual se debería mantener un caudal mínimo y realizar su medición, así como para establecer cuál es el contenido mínimo que debería tener un estudio para determinar el caudal ecológico. ${ }^{11}$
En ese sentido, es claro que la aplicación de la Metodología en estos casos concretos es en todo extremo incorrecta e ilegal, y llega a ser, inclusive, inconstitucional pues vulnera principios reconocidos expresamente en nuestra Constitución como el de legalidad e irretroactividad (Ventura, 2017).

Asimismo, cabe precisar que la determinación de los caudales ecológicos que realice la ANA en coordinación con el Ministerio del Ambiente para la ejecución de obras de infraestructura hidráulica, deberá tomar en cuenta los derechos de uso de agua preexistentes en cada fuente natural e, incluso, aquellas autorizaciones preexistentes, con el objeto de aprovechar un determinado volumen de agua.

Siendo este el caso, afirmar lo contrario podría llevarnos hasta un supuesto de expropiación indirecta que afectaría la seguridad jurídica de los titulares de actividades que se desarrollen en diferentes fuentes de agua natural en virtud de derechos de agua previamente otorgados y válidamente obtenidos (Ventura, 2017).

Sobre el particular, el artículo 4 del Título Preliminar de la LRH que regula el principio de seguridad jurídica, señala que “(e)l Estado consagra un régimen de derechos para el uso del agua. Promueve y vela por el respeto de las condiciones que otorgan seguridad jurídica a la inversión relacionada con su uso, sea pública o privada o en coparticipación".

Por todo lo antes expuesto, consideramos que no correspondía que el OEFA pretenda aplicar el concepto de caudal ecológico regulado en las normas vigentes en materia de recursos hídricos en los procedimientos administrativos sancionadores seguidos con los Expedientes 615-2013-OEFA/DFSAI/ PAS y 598-2014-OEFA/DFSAI/PAS, pues este no les resultaba exigible.

Si bien el Tribunal de Fiscalización Ambiental (en adelante, "TFA") -segunda y última instancia administrativa del OEFA- en las resoluciones emitidas en el marco de los procedimientos administrativos sancionadores antes mencionados ha revocado los pronunciamientos de la DFAI en primera instancia, no ha llegado a pronunciarse sobre la aplicación referencial de la LRH y su Reglamento ni de la Metodología en relación al concepto de caudal ecológico. Por el contrario, se ha limitado

11 A través del Decreto Supremo 017-2017-MINAM, publicado con fecha 21 de diciembre de 2017 en el Diario Oficial EI Peruano, el Ministerio del Ambiente aprobó el nuevo Reglamento de Organización y Funciones del OEFA. Mediante dicha norma, la Dirección de Fiscalización, Sanción y Aplicación de Incentivos pasó a denominarse Dirección de Fiscalización y Aplicación de Incentivos. Asimismo, la Subdirección de Instrucción e Investigación fue reemplazada por la Subdirección de Fiscalización en Energía y Minas (en el marco de procedimientos administrativos sancionadores seguidos contra titulares de actividades mineras y energéticas). 
a realizar una interpretación literal de los compromisos ambientales asumidos por ambas empresas hidroeléctricas en sus IGA y ha concluido que el modo de cumplimiento de los mismos exigido por el OEFA no se desprende de dichos compromisos.

En ese contexto, aunque el TFA no es la autoridad competente para determinar criterios sobre el manejo de los recursos hídricos, su falta de pronunciamiento sobre este tema ha generado que todavía continúe latente la posibilidad de que en un procedimiento administrativo sancionador posterior el OEFA vuelva a tratar de aplicar el concepto vigente de caudal ecológico a titulares de proyectos hidroenergéticos que han iniciado sus actividades con anterioridad a su existencia.

Lo anterior continuará siendo de esta manera hasta que la ANA, como ente rector y máxima autoridad en materia hídrica, emita un pronunciamiento formal que reafirme lo regulado en la Metodología y señale que cualquier norma emitida en relación al concepto de caudal ecológico no se aplica retroactivamente ni afecta el ejercicio de los derechos de uso de agua otorgados con anterioridad ni las acreditaciones de disponibilidad hídrica, o los caudales ecológicos aprobados antes de su entrada en vigencia.

Finalmente, cabe señalar que, mediante Resolución Jefatural 212-2017-ANA, publicada en el Diario Oficial El Peruano con fecha 29 de agosto de 2017, la ANA ha pre publicado el proyecto de Lineamientos para determinar caudales ecológicos que, de ser aprobado, derogará la Metodología.

Sin perjuicio de las modificaciones que establece este proyecto para la determinación del caudal ecológico, resulta relevante resaltar que sigue la misma lógica de la Metodología al establecer que los caudales ecológicos determinados en base a esta norma no afectan el ejercicio de los derechos de uso de agua otorgados, las acreditaciones de disponibilidad hídrica, o los caudales ecológicos aprobados antes de su entrada en vigencia.

Siendo ello así, los titulares de proyectos hidroenergéticos pueden tener la certeza de que -en caso se apruebe dicha norma- no se variarán las reglas de juego en ese extremo ni se admitirán modificaciones en las condiciones de derechos de uso de agua previamente otorgados.

\section{Sobre la aplicación del Plan de Aprovecha- miento de las Disponibilidades Hídricas}

La primera referencia que se realiza en las normas vigentes en materia de recursos hídricos al Plan de Aprovechamiento de las Disponibilidades Hídricas (en adelante, "PADH") se encuentra en el Reglamento.

El artículo 31 de dicha norma señala que es una función del Consejo de Recursos Hídricos de Cuenca proponer anualmente a la Autoridad Administrativa del Agua (en adelante, "AAA"), organismo desconcentrado de la ANA, el PADH para atender las demandas multisectoriales, "considerando los derechos de uso de agua otorgados y usos de agua de las comunidades campesinas y comunidades nativas" cuando se encuentren dentro del ámbito de este órgano.

Posteriormente, cinco (5) años luego de la entrada en vigencia del Reglamento, mediante Resolución Jefatural 315-2014-ANA, publicada con fecha 21 de noviembre de 2014 en el Diario Oficial El Peruano, se aprobó el Reglamento del Plan de Aprovechamiento de las Disponibilidades Hídricas (en adelante, el "Reglamento del PADH"), es decir, la primera norma que estableció qué aspectos comprendía el PADH y cómo debía darse el procedimiento administrativo para su elaboración y aprobación, incluyendo qué entidades participaban en el mismo. ${ }^{12}$

El numeral 5.2 del artículo 5 del Reglamento del PADH lo definió como:

"un instrumento técnico vinculante del Plan de Gestión de Recursos Hídricos en la Cuenca, que permite la planificación anual, uso multisectorial y conjunto (superficial, subterránea, residual) de la disponibilidad de agua para atender la demanda multisectorial de los derechos de uso de agua otorgados y del caudal ecológico; teniendo en cuenta el comportamiento hidrológico y climatológico".

Posteriormente, el artículo 28 de la Resolución Jefatural 327-2018-ANA, que aprobó el Reglamento de Operadores de Infraestructura Hidráulica (en adelante, el "Reglamento de Operadores") -y derogó el Reglamento del PADH- estableció que este

12 A la fecha, la Resolución Jefatural 315-2014-ANA ha sido derogada por la Resolución Jefatural 327-2018-ANA, que aprobó el Reglamento de Operadores de Infraestructura Hidráulica, publicada con fecha 1 de noviembre de 2018 en el Diario Oficial El Peruano. 
"Es un instrumento de planificación anual del uso multisectorial de los recursos hídricos para atender las demandas de agua de los titulares de derechos de uso de agua, caudal ecológico, la seguridad de la presa y evitar el riesgo de las poblaciones, actividades económica e infraestructura pública y privada ubicadas aguas debajo de la presa o cercanas al espejo de agua y otros. Para su elaboración se tiene en cuenta el comportamiento hidrológico, climatológico, socio-ambiental y las características de la infraestructura hidráulica".

En ese contexto, considerando que el Plan de Gestión de los Recursos Hídricos en la Cuenca es a su vez un instrumento técnico de carácter público y vinculante que tiene por finalidad alcanzar el uso sostenible de los recursos hídricos, la finalidad principal del Reglamento del PADH era proporcionar a los Consejos de Recursos Hídricos de Cuenca una herramienta que permita planificar anualmente el uso multisectorial y conjunto de la disponibilidad hídrica, siempre considerando el comportamiento hidrológico y las demandas de agua de acuerdo con los derechos de uso de agua otorgados. Cabe resaltar que la finalidad antes mencionada se mantuvo luego de la derogación del Reglamento del PADH, de conformidad con el Reglamento de Operadores.

Siendo ello así, el PADH tiene como objetivo atender las demandas multisectoriales, pero sin afectar los derechos de uso de agua previamente otorgados. En otras palabras, los PADH que sean aprobados no podrían -en ningún caso- modificar los términos y condiciones de derechos de uso de agua otorgados de manera previa, los cuales deben ser considerados por la autoridad competente al momento del análisis para su elaboración de modo que se logre una adecuada planificación que se encuentre de acuerdo con la disponibilidad hídrica existente en determinado sector.

No obstante, -en la práctica y durante la vigencia del Reglamento del PADH- algunas AAA han elaborado y aprobado PADH sin considerar los derechos de uso de agua existentes previamente y en algunos casos incluso generando afectaciones a los mismos e impidiendo que cumplan el fin para el cual han sido otorgados por la autoridad de aguas competente.

Esto último no solo contradice la naturaleza del PADH según lo señalado por las normas vigentes en la materia, sino que implicaría una vulneración al principio de seguridad jurídica recogido en el numeral 4 del artículo III del Título Preliminar de la LRH, el cual señala que "el Estado consagra un régimen de derechos para el uso del agua y al mismo tiempo promueve y vela por el respeto de las condiciones que otorgan seguridad jurídica a la inversión relacionada con su uso, sea pública o privada o en coparticipación".

Asimismo, cabe resaltar que el principio descrito se ve reflejado en el artículo 46 de este dispositivo que establece que "se encuentra prohibido alterar, modificar, perturbar o impedir el uso legítimo del agua". En esa línea, se señala que es un deber del Estado garantizar el cumplimiento de los derechos de uso otorgados.

Por este motivo, un instrumento técnico y vinculante como el PADH no podría afectar de ninguna manera derechos de uso de agua que hayan sido otorgados previamente a su elaboración y/o aprobación, ni debería generar restricciones a sus titulares.

Sobre el particular, un precedente que es importante tomar en cuenta es el pronunciamiento del Tribunal Nacional de Resolución de Controversias Hídricas (en adelante, "TNRCH") de la ANA en el caso del PADH del río Santa aprobado por la AAA Huarmey Chicama para el año 2014.

Al respecto, en virtud de un recurso de apelación interpuesto por una empresa hidroeléctrica contra el acto administrativo que aprobó el PADH del río Santa para el año 2014, el TNRCH emitió la Resolución 418-2015-ANA/TNRCH, de fecha 6 de julio de 2015, en la cual reconoce de manera expresa que las medidas incluidas por dicha autoridad en el PADH antes descrito vulneraban y/o afectaban los derechos de uso de agua otorgados previamente a favor de dicha empresa para la operación de su proyecto hidroenergético impidiendo la adecuada ejecución de sus actividades de generación eléctrica.

En ese sentido, tras la emisión de este precedente por parte del TNRCH, la AAA Huarmey Chicama no podría incluir en un nuevo PADH aprobado para futuros períodos medidas restrictivas contra derechos de uso de agua previamente otorgados a terceros. Ello también resultaría aplicable para otras AAA que se encuentren elaborando un PADH para su ámbito de competencia.

Sin perjuicio de lo antes expuesto, un aspecto que también resulta relevante mencionar en relación a los PADH es que el Reglamento del PADH al regular el procedimiento para la elaboración y posterior aprobación de dicho instrumento técnico no había brindado las herramientas idóneas a los terceros titulares de derechos de uso de agua con sistemas de abastecimiento de agua propios para que puedan garantizar la debida consideración de dichos 
derechos previamente otorgados en la propuesta de PADH que será aprobado.

Ello se evidencia al analizar las etapas comprendidas durante el procedimiento para la formulación y aprobación del PADH correspondiente según su Reglamento, pues los titulares de derechos de uso de agua preexistentes con sistemas de abastecimiento de agua propios no participaban directamente.

\section{Resolución Jefatural 315-2014-ANA, Reglamento del Plan de Aprovechamiento de las Disponibilidades Hídricas}

FLUJOGRAMA-PROCEDIMIENTO DE APROBACIÓN

DEL PLAN DE APROVECHAMIENTO

DE LAS DISPONIBILIDADES HÍDRICAS
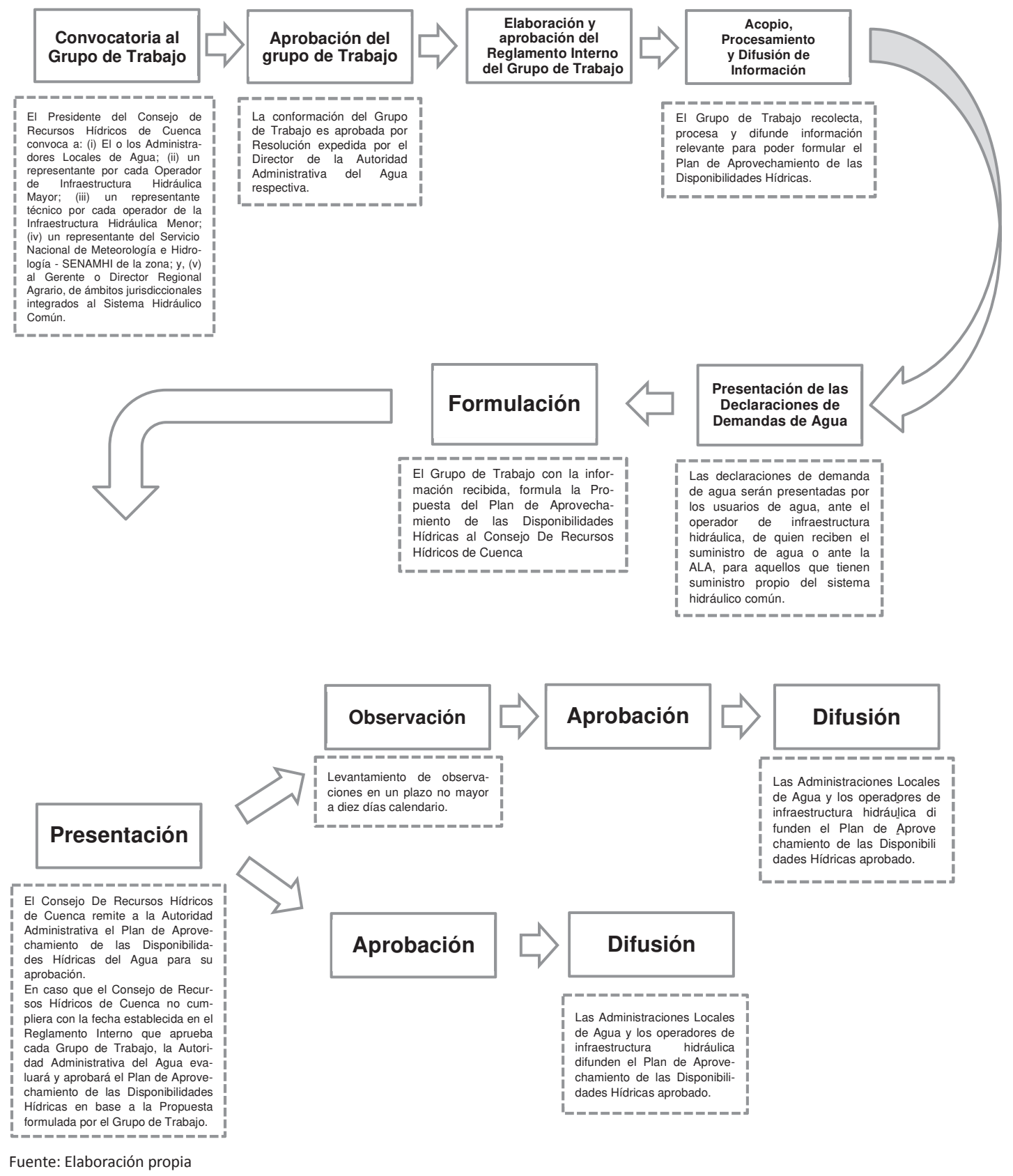
El numeral 11.2 del artículo 11 del Reglamento del PADH señalaba expresamente que los usuarios con sistemas de abastecimiento de agua propios solo tenían como responsabilidades: (i) remitir su demanda de agua a la Autoridad Local del Agua (en adelante, "ALA") competente a través del Formulario PADH-2 "Demanda de Agua del Usuario", aprobado mediante dicha norma; y, (ii) solicitar la modificación de la demanda de agua a la ALA, de ser el caso.

Si bien en esa línea el numeral 34.2 del artículo 34 del Reglamento de Operadores establece que los usuarios de agua con sistemas propios presentan la demanda de agua, utilizando el Formato E-5 del Anexo E, a más tardar el 31 de mayo ante la ALA. La principal diferencia con el Reglamento del PADH es que, en virtud de la nueva regulación, estos titulares de derechos de uso de agua sí podrán ser considerados durante el procedimiento como parte del Grupo de Trabajo conformado por el Consejo de Recursos Hídricos de Cuenca que tiene como finalidad la formulación, aprobación, ejecución, seguimiento y supervisión del PADH.

Según el artículo 29 del Reglamento de Operadores, este grupo se encuentra compuesto por:

(i) el Secretario Técnico del Consejo de Recursos Hídricos de Cuenca, quien la preside;

(ii) el o los Administradores Locales de Agua;

(iii) un representante por cada Operador de Infraestructura Hidráulica Mayor;

(iv) un representante por cada Operador de la Infraestructura Hidráulica Menor;

(v) Gerente o Director Regional Agrario, de ámbitos jurisdiccionales integrados al Sistema Hidráulico Común;

(vi) un representante del Ministerio de Agricultura y Riego;

(vii) un representante del Servicio Nacional de Meteorología e Hidrología - SENAMHI de la zona; en donde exista; y,

(viii) en sistemas hidráulicos comunes donde exista un usuario con su propio sistema de abastecimiento de agua con obras de regulación podrá integrarse al grupo [El énfasis es nuestro].

Siendo este el caso, otra distinción entre el Reglamento del PADH y el Reglamento de Operadores, es que dicha norma ha variado significativamente el procedimiento de elaboración del PADH por parte del Grupo de Trabajo, como puede observarse en el flujograma presentado en la página siguiente.

En vista de ello, anteriormente los usuarios con sistemas de abastecimiento de agua propios no contaban con la posibilidad de participar en las reunio- nes celebradas por el Grupo de Trabajo donde se discutía cuál sería el contenido de la propuesta del PADH ni tampoco tenían un representante específico. En otras palabras, su única forma de participación durante el procedimiento de formulación del PADH era a través de la remisión de información a la ALA competente.

Así, esta -prácticamente- nula participación durante el procedimiento regulado por el Reglamento del PADH no permitía a los titulares de derechos de uso de agua preexistentes exponer adecuadamente su posición y situación ante la futura aprobación de un PADH que podría restringir y/o afectar estos derechos.

Desde nuestro punto de vista, a efectos de mejorar el procedimiento elaboración y aprobación del PADH para evitar que se aprueben dichos instrumentos técnicos sin considerar los derechos de uso de agua previamente otorgados y garantizar de esta manera el principio de seguridad jurídica contemplado en la LRH, era necesario que los titulares de derechos de uso de agua con sistemas de abastecimiento de agua propios puedan participar activamente en las reuniones llevadas a cabo por el Grupo de Trabajo a cargo de la formulación de la propuesta de $\mathrm{PADH}$, lo cual ha sido incorporado mediante el Reglamento de Operadores.

Es importante tomar en cuenta que, previamente a la aprobación del Reglamento de Operadores, mediante Resolución Jefatural 230-2017-ANA, publicada con fecha 20 de setiembre de 2017 en el Diario Oficial El Peruano, se dispuso la prepublicación del proyecto de Reglamento de Operadores de Infraestructura Hidráulica y de los Instrumentos para la prestación de los servicios de suministro de agua en el portal web de la ANA (en adelante, el Proyecto).

Mediante el Proyecto, la ANA buscó regular la prestación de los servicios públicos de suministro de agua superficial y de monitoreo y gestión de aguas subterráneas, así como el contenido, aprobación y supervisión de los instrumentos técnicos que presentan los operadores de infraestructura hidráulica y usuarios con sistema propio de abastecimiento de agua.

Entre otras disposiciones, el Proyecto establecía las atribuciones y responsabilidades del operador de infraestructura hidráulica para prestar los servicios antes mencionados, así como los siguientes instrumentos técnicos con los que cuenta: (i) Padrón de Usuarios del servicio en concordancia con el Registro Administrativo de Derechos de Uso de Agua; (ii) Inventario de Infraestructura Hidráulica; (iii) Plan Multianual de Inversiones; (iv) Plan 


\section{FLUJOGRAMA PROCEDIMIENTO DE APROBACIÓN DEL PLAN DE APROVECHAMIENTO DE LAS DISPONIBILIDADES HÍDRICAS}

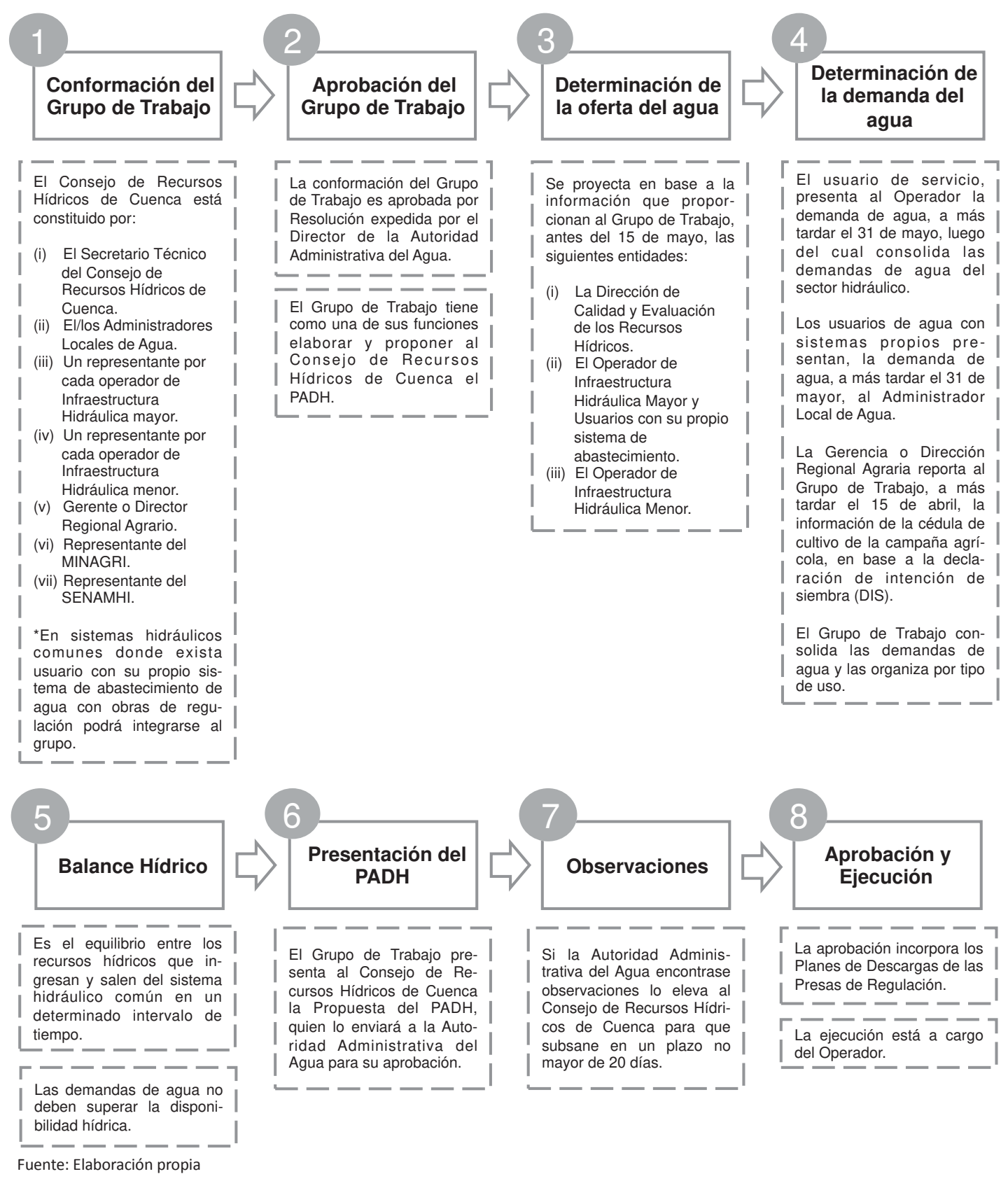

de Operación, Mantenimiento y Desarrollo de Infraestructura Hidráulica; (v) PADH; y, (vi) Planes de Descarga de Embalses. El detalle del contenido de dichos instrumentos se encuentra en los Anexos que forman parte del Proyecto.

Al respecto, en relación al $\mathrm{PADH}$, mediante el Proyecto se reguló con mayor detalle el proceso para su elaboración, inclusive señalando fechas específicas en las que se debía remitir al Grupo de Trabajo la información respectiva para determinar la oferta y demanda de agua. Respecto a los usuarios de agua con sistemas propios, se estableció que estos debían presentar la demanda de agua a más tardar el 31 de mayo a la ALA competente. No obstante, el Proyecto tampoco hacía mayor 
referencia a su participación durante el proceso de elaboración del PADH y se limitaba a que esta sea la única información que remitan y que pueda ser tomada en cuenta para la implementación de dicho instrumento.

Tomando en cuenta que finalmente el Reglamento de Operadores sí consideró una referencia a la participación más activa de los titulares de proyectos hidroenergéticos con sistemas de abastecimiento de agua propios durante la formulación del PADH al incorporarlos en el Grupo de Trabajo, resulta probable que dichos titulares hayan remitido comentarios y aportes al Proyecto de modo que pudieran ser tomados en cuenta durante dicho procedimiento.

Sin perjuicio de lo anterior, consideramos que lo mencionado no debería implicar que todos los titulares de derechos de uso de agua con sistemas de abastecimiento de agua propios formen parte del Grupo de Trabajo conformado por el Consejo de Recursos Hídricos de Cuenca, pues esto resultaría claramente inviable debido a la cantidad de usuarios que podría existir dependiendo del sector hidráulico.

La participación que sugerimos es una participación con voz pero sin voto durante las reuniones celebradas por el Grupo de Trabajo, la cual permitiría únicamente garantizar que el futuro PADH que será aprobado por la AAA no genere algún tipo de afectación y/o restricción a derechos de uso de agua ya existentes que podría impedir el normal desarrollo de las actividades para los cuales fueron otorgados.

En síntesis, consideramos que el PADH es una herramienta técnica que resulta de utilidad para realizar una adecuada planificación del uso de la disponibilidad de agua existente en determinado sector hidráulico, de modo que pueda atenderse la demanda de los usuarios.

No obstante, a efectos de garantizar el adecuado cumplimiento de sus fines, resulta necesario que las AAA tomen en cuenta todos los derechos de uso de agua existentes en los sectores hidráulicos a su cargo para no aprobar un PADH que sea restrictivo ni afecte estos derechos. Asimismo, esperamos que el Reglamento de Operadores permita otorgarles una mayor y más activa participación a los titulares de derechos de uso de agua con sistemas de abastecimiento de agua propios durante el procedimiento de formulación del respectivo PADH para que sea posible realizar un análisis más detallado de su situación ante la aprobación de dicho instrumento.

\section{CONCLUSIONES}

Como se ha señalado a lo largo del presente artículo, los recursos hídricos son un elemento esencial para la ejecución de la actividad hidroenergética. Siendo este el caso, el titular de un proyecto hidroenergético tiene que cumplir con diversos procedimientos, así como con una serie de obligaciones establecidas en la normativa vigente en materia de aguas con la finalidad de poner en marcha y operar dicho proyecto.

Ante tal escenario, el Estado -a través de la ANA como órgano rector de los recursos hídricos en el Perú- debería garantizar que existan criterios claros y definidos que permitan a los titulares de proyectos hidroeléctricos contar con las facilidades necesarias para ejecutar sus actividades con regularidad.

No obstante, de la revisión de la legislación vigente y las actuaciones de las autoridades competentes se han identificado algunos aspectos cuestionables que generan trabas e impiden un adecuado desarrollo de los proyectos hidroenégeticos como son principalmente los siguientes

(i) No existe una definición única del término "aguas turbinadas", lo cual genera que sean categorizadas como efluentes líquidos por algunas autoridades con competencias en materia de fiscalización ambiental como el OEFA. Esto último podría producir confusiones a los titulares de proyectos hidroenergéticos respecto a las obligaciones ambientales que les resultarían aplicables en relación a dichas aguas (i.e. la obtención de una autorización de vertimiento de aguas residuales tratadas; la ejecución de muestreos y análisis químicos mensuales, entre otras).

(ii) Si bien la normativa vigente -principalmente la Metodología- ha regulado el concepto de caudal ecológico como aquel volumen de agua que se debe mantener en las fuentes naturales de agua para la protección o conservación de los ecosistemas involucrados, la estética del paisaje u otros aspectos de interés científico o cultural, estableciendo que este no se aplica retroactivamente ni afecta el ejercicio de los derechos de uso de agua otorgados con anterioridad ni las acreditaciones de disponibilidad hídrica, o los caudales ecológicos aprobados antes de su entrada en vigencia; autoridades con competencia en materia de fiscalización ambiental como el OEFA han tratado de aplicar el concepto vigente de caudal ecológico a titulares de proyectos hidroenergéticos que han 
iniciado sus actividades con anterioridad a su existencia. A la fecha, no se cuenta con un pronunciamiento formal de la ANA como ente rector y máxima autoridad en materia hídrica que precise este tema y reafirme lo señalado en la Metodología.

(iii) Durante el procedimiento de elaboración y aprobación de los PADH, los cuales son herramientas técnicas que resultan de utilidad para realizar una adecuada planificación del uso de la disponibilidad de agua existente en determinado sector hidráulico de modo que pueda atenderse la demanda de los usuarios, las AAA, en ocasiones, no toman en cuenta todos los derechos de uso de agua existentes en los sectores hidráulicos a su cargo, lo cual podría generar la aprobación de PADH que sean restrictivos y afecten estos derechos. A efectos de evitar lo anterior, resultaba necesario que la normativa vigente les otorgue una mayor y más activa participación a los titulares de derechos de uso de agua con sistemas de abastecimiento de agua propios durante el procedimiento de formulación del respectivo PADH. Esto último parece finalmente haber sido adoptado mediante el nuevo Reglamento de Operadores.

En síntesis, consideramos que para solucionar la problemática descrita en párrafos anteriores, sería recomendable que se emitan dispositivos legales que uniformicen los criterios establecidos en la normativa vigente en materia de recursos hídricos y precisen aquellos aspectos que se encuentran poco claros. De esta manera, se brindará a las autoridades competentes mayores herramientas que permitan evitar contradicciones en sus pronunciamientos. 疅

\section{REFERENCIAS}

Licla Tomayro, L. (2012). "Historia de la electricidad en el Perú". Energía Hídrica - La energía tiene tal vitalidad, que sobrevive a todos los desastres y provee a todos los esfuerzos. Recuperado de: http://hidricae.blogspot. pe/2012/06/historia-de-la-electricidad-enel-peru.html

Organismo de Evaluación y Fiscalización Ambiental (2016). Criterios sustantivos emitidos en el subsector electricidad. Principales criterios resolutivos adoptados en los procedimientos administrativos sancionadores del Organismo de Evaluación y Fiscalización Ambiental (OEFA). Compilación 2011-2015 (pp. 174178). Lima: OEFA.
Ventura Rivas Plata, A. (2017). “OEFA debe sentar criterios claros para garantizar seguridad jurídica de los principales proyectos hidroeléctricos del país". Miranda \& Amado Abogados. Recuperado de http://mafirma. pe/?publicacion=oefa-debe-sentar-criteriosclaros-para-garantizar-seguridad-juridicade-los-principales-proyectos-hidroelectricos-del-pais

\section{LEGISLACIÓN, JURISPRUDENCIA Y OTROS DO- CUMENTOS LEGALES}

Decreto Ley 25844. Ley de Concesiones Eléctricas. En: Diario El Peruano, 19 de noviembre de 1992.

Decreto Ley 17752. La Ley General de Aguas. En: Diario El Peruano, 24 de julio de 1969.

Decreto Supremo 001-2010-AG. Reglamento de la Ley 29338, Ley de Recursos Hídricos. En: Diario El Peruano, 24 de marzo de 2010.

Ley 29338. Ley de Recursos Hídricos. En: Diario El Peruano, 31 de marzo de 2009.

Oficio 513-2013-ANA-DGCRH. 28 de agosto de 2013.

Resolución 418-2015-ANA/TNRCH, recaída en el Expediente 72550-2014. En: Portal Institucional de la ANA, 6 de julio de 2015.

Resolución Directoral 018-2017-OEFA/TFA-SMEPIM, recaída en el Expediente 615-2013-OEFADFSAI/PAS. En: Portal de Transparencia del OEFA, 6 de julio de 2017.

Resolución Directoral 017-2017-OEFA/TFA-SMEPIM, recaída en el Expediente 598-2014-OEFADFSAI/PAS. En: Portal de Transparencia del OEFA, 22 de junio de 2017.

Resolución Directoral 008-97-EM/DGAA. Niveles máximos permisibles para efluentes líquidos producto de las actividades de generación, transmisión y distribución de energía eléctrica. En: Diario El Peruano, 17 de marzo de 1997.

Resolución Jefatural 327-2018-ANA. Reglamento de Operadores de Infraestructura Hidráulica. En: Diario El Peruano, 1 de noviembre de 2018.

Resolución Jefatural 230-2017-ANA. Proyecto de Reglamento de Operadores de Infraestruc- 
tura Hidráulica y de los Instrumentos para la prestación de los servicios de suministro de agua. En: Diario El Peruano, 20 de setiembre de 2017.

Resolución Jefatural 212-2017-ANA. Proyecto de Lineamientos Generales para determinar Caudales Ecológicos. En: Diario El Peruano, 29 de agosto de 2017.

Resolución Jefatural 180-2016-ANA. Glosario de Términos sobre Recursos Hídricos. En: Portal Institucional del ANA, 07 de julio de 2016.
Resolución Jefatural 154-2016-ANA. Metodología para Determinar Caudales Ecológicos. En: Diario El Peruano, 17 de junio de 2016.

Resolución Jefatural 315-2014-ANA, Reglamento del Plan de Aprovechamiento de las Disponibilidades Hídricas. En: Diario El Peruano, 21 de noviembre de 2014.

Resolución Ministerial 031-2018-MEM/DM. Proyecto de Reglamento de Protección Ambiental en las Actividades Eléctricas. En: Diario El Peruano, 25 de enero de 2018. 